\title{
Renal-targeted delivery of triptolide by entrapment in pegylated TRX-20-modified liposomes
}

This article was published in the following Dove Press journal:

International Journal of Nanomedicine

8 August 2017

Number of times this article has been viewed

\author{
Zhi-xiang Yuan ${ }^{1, *}$ \\ Lu Jia ${ }^{2, *}$ \\ Lee Yong $\mathrm{Lim}^{3}$ \\ Ju-chun Lin' \\ Gang Shu' \\ Ling Zhao' \\ Gang $\mathrm{Ye}^{\prime}$ \\ Xiao-xia Liang' \\ Hongming $\mathrm{Ji}^{2}$ \\ Hua-lin $\mathrm{Fu}^{\prime}$ \\ 'Department of Pharmacy, \\ College of Veterinary Medicine, \\ Sichuan Agricultural University, \\ Chengdu, Sichuan, ${ }^{2}$ Department of \\ Neurosurgery, Shanxi Provincial \\ People' Hospital, Taiyuan, China; \\ ${ }^{3}$ Pharmacy, Centre for Optimization \\ of Medicines, School of Allied Health, \\ The University of Western Australia, \\ Crawley, Australia \\ *These authors contributed equally \\ to this work
}

Correspondence: Hongming Ji

Department of Neurosurgery, Shanxi

Provincial People' Hospital, No 29,

Shuangtasi Road, Taiyuan, Shanxi 030012 ,

China

Tel +86 35I 4960343

Email hongmingj@sina.com

Hua-lin Fu

Department of Pharmacy, College of

Veterinary Medicine, Sichuan Agricultural

University, No 21I, Huimin Road,

Shuangliu District, Chengdu, Sichuan

611130, China

Tel +86 I3308I6 28I6

Email fuhl2005@sohu.com
Abstract: Previously, 3,5-dipentadecyloxybenzamidine hydrochloride (TRX-20)-modified liposomes were reported to specifically target mesangial cells (MCs) in glomeruli. To further gain a better understanding of the characteristics and potential application for glomerular diseases of TRX-20-modified liposomes, we synthesized TRX-20 and prepared TRX-20-modified liposomes (TRX-LPs) with different molar ratios - 6\% (6\%-TRX-LP), 11\% (11\%-TRX-LP), and 14\% (14\%-TRX-LP) - of TRX-20 to total lipid in the present study. All TRX-LPs exhibited concentration-dependent toxicity against the MCs at a lipid concentration ranging from 0.01 to $1.0 \mathrm{mg} / \mathrm{mL}$ with $\mathrm{IC}_{50}$ values of $3.45,1.13$, and $0.55 \mathrm{mg} / \mathrm{mL}$, respectively. Comparison of the cell viability of TRX-LPs indicated that high levels of TRX-20 caused severe cell mortality, with $11 \%$-TRX-LP showing the higher cytoplasmic accumulation in the MCs. Triptolide (TP) as a model drug was first loaded into 11\%-TRX-LP and the liposomes were further modified with PEG $_{5000}$ (PEG-TRX-TP-LP) in an attempt to prolong their circulation in blood and enhance TP-mediated immune suppression. Due to specific binding to MCs, PEG-TRX-TP-LP undoubtedly showed better anti-inflammatory action in vitro, evidenced by the inhibition of release of nitric oxide (NO) and tumor necrosis factor- $\alpha$ from lipopolysaccharide-stimulated MCs, compared with free TP at the same dose. In vivo, the PEG-TRX-TP-LP effectively attenuated the symptoms of membranous nephropathic (MN) rats and improved biochemical markers including proteinuria, serum cholesterol, and albumin. Therefore, it can be concluded that the TRX-modified liposome is an effective platform to target the delivery of TP to glomeruli for the treatment of MN.

Keywords: triptolide, pegylated liposomes, TRX-20, renal targeting, mesangial cells

\section{Introduction}

The kidneys are vital organs of humoral regulation, and they maintain the homeostasis of nutrients and metabolites in the body. Renal dysfunction may lead to chronic kidney disease, generally acknowledged as a serious problem affecting public health ${ }^{1}$ as it eventually requires expensive and debilitating renal replacement therapy, such as dialysis or renal transplantation, to sustain the lives of patients. Drug therapies of renal diseases often induce complicated extrarenal toxicity. Selective targeting of drugs to kidneys can realize an increased renal effectiveness combined with a reduction of generalized side effects. ${ }^{2-4}$ Targeted therapies can also lower drug doses to further minimize side effects, an especially attractive option for chronic renal diseases requiring treatment over extended periods. ${ }^{5}$

Renal drug targeting could be directed at the renal tubules or glomeruli depending on the diseases to be treated. In previous studies, we have developed several drug carriers 
with capacity for renal tubular targeting, including lowmolecular-weight chitosans, ${ }^{6,7}$ glucosamines, ${ }^{8,9}$ and peptide fragments of human serum albumin. ${ }^{10,11}$ These carriers have been shown to achieve effective targeted delivery of prednisolone or triptolide (TP) to the renal tubules. However, they may not be effective carriers of drugs required for the treatment of inflammatory and pathogenic glomeruli diseases.

In recent years, a great many studies have demonstrated a key point for glomerular cell responses in the progression of renal disease, which enlightens us that drug delivery to the glomerulus restraining local inflammatory/pathological reactions could be hoped to produce better therapeutic results., ${ }^{5,12}$ Tuffin et al first proposed immunoliposomes as a glomerular delivery system for the treatment of kidney diseases gradually. ${ }^{13}$ They developed OX7-coupled immunoliposomes by coupling liposomes with $\mathrm{F}_{\mathrm{ab}}$, fragments of $\mathrm{OX} 7$ monoclonal antibody directed against Thy1.1 antigen, which is specifically expressed in mesangial cells (MCs). Intravenous injection of OX-7-IL to rats showed a specific targeting of all MCs in both kidneys. ${ }^{13}$ Due to the strong and exclusive expression of $\alpha 8$ integrin in $\mathrm{MCs},{ }^{14}$ Scindia et al selected anti- $\alpha 8$ integrin as a ligand to prepare immunoliposomes. Their findings demonstrated the specific delivery of anti- $\alpha 8$ integrin immunoliposomes to the mesangium following tail vein injection in mice. ${ }^{15}$ Moreover, Morimoto et al developed novel glomeruli-targeting liposome co-modified by 3,5-dipentadecyloxybenzamidine hydrochloride (TRX-20) and PEG $_{5000}$. PEGylation can prolong blood circulation time of the liposomes and allowed them to accumulate in targeting tissues where the liposomes repeatedly pass by. The final liposomes have diameter of around $100 \mathrm{~nm},{ }^{16}$ positive zeta potential, and high selection for MCs over vascular endothelial cells. ${ }^{17}$

It is clear that MCs as an effective target site are adopted in all the studies mentioned above. MCs comprise $\sim 30 \%-40 \%$ of the total glomerular cell population, and they play a central role in maintaining the structure and regulating the surface area of the glomerular filtration barrier by virtue of their contractile capacities. ${ }^{13}$ MCs are often involved in multiple biological responses elicited by circulating factors and metabolites, including cytoproliferation, apoptosis, cellular migration, and the elaboration of reactive oxygen species, cytokines, and chemokines, which undergird many glomerular diseases. Therefore, the selective delivery of drugs to glomerular MCs could considerably improve the therapeutic outcome of immunoglobulin A nephropathy and other glomerulopathies than targeting drug delivery to the renal tubular cells.
To gain a better understanding of the characteristics and potential applications of glomerular diseases of TRX-20modified liposomes (TRX-LPs), we synthesized TRX-20 and prepared TRX-LPs with different molar ratios of TRX-20 to total lipid in this study. Cytotoxicity of TRX-LPs toward the MCs was performed for the first time. The binding affinities to MCs of liposomes modified with different TRX-20 molar ratios and loaded with a fluorescent agent were evaluated by laser confocal microscopy. Finally, TP as a model drug was first loaded into TRX-LP with PEG $_{5000}$ co-modification (PEG-TRX-TP-LP) in an attempt to enhance TP-mediated immune suppression. In vitro anti-inflammatory activity was determined by measuring the nitric oxide (NO) and tumor necrosis factor- $\alpha$ (TNF- $\alpha$ ) released from lipopolysaccharide (LPS)-stimulated MCs. Additionally, the efficacy of PEG-TRX-TP-LP was evaluated in vivo in a membranous nephropathy $(\mathrm{MN})$ rat model.

\section{Materials and methods Materials and animals}

3,5-dihydroxybenzonitrile, 1-bromopentadecane, cholesterol, collagenase IV, Coumarin-6 (C6), and pluronic 188 (F68) were from Sigma-Aldrich Co. Ltd. (Gillingham, UK). TP was purchased from Xieli Biotechnology Co. Ltd. (Sichuan, China). Hydrogenated soybean phosphatidylcholine (HSPC) and polyethylene glycol 5000-sn-glycrero-3phosphatydilethanolamine $\left(\mathrm{PEG}_{5000}-\mathrm{PE}\right)$ were obtained from Lipoid Co. Ltd. (Ludwigshafen, Germany). 4',6-diamidino2-phenylindole dihydrochloride (DAPI) was supplied by Roche Co. Ltd. (Shanghai, China). Fetal bovine serum and RPMI 1640 were purchased from Thermo Scientific Co. Ltd. (IL, USA). All other chemicals and solvents were of the analytical grade.

Sprague Dawley (SD) rats (200 \pm 20 g, male, 6 weeks old) were obtained from the Da-Shuo Experimental Animal Ltd. (Beijing, China). The animals were allowed to acclimatize for a few days in environmentally controlled quarters $\left(24^{\circ} \mathrm{C} \pm 1^{\circ} \mathrm{C}\right.$, $12 \mathrm{~h}$ light/dark cycle) and, unless specified otherwise, were provided with water and normal diets ad libitum. All animal studies were approved by the Animal Ethical Experimentation Committee of Sichuan Academy of Chinese Medicine Sciences (SYXK[Chuan]2013-100), and were performed according to the requirements of the People's Republic of China National Act on the use of experimental animals.

\section{Synthesis of TRX-20}

The synthesis of TRX-20 was carried out according to Harigai et al's method with some improvements ${ }^{17}$ (Figure 1). 

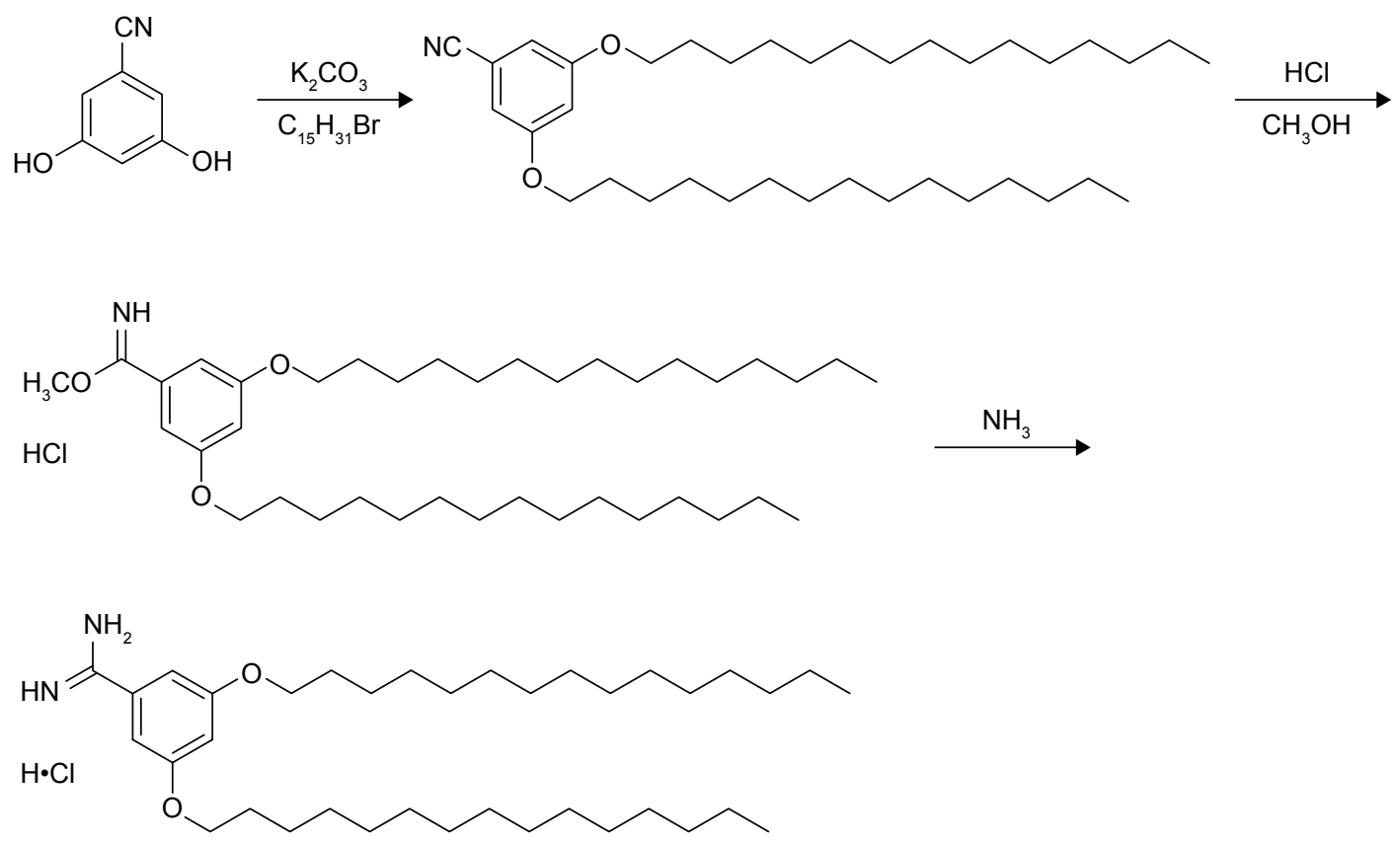

Figure I Synthetic route of 3,5-dipentadecyloxybenzamidine hydrochloride.

3,5-dihydroxybenzonitrile (0.405 g), 1-bromopentadecane $(1.85 \mathrm{~g})$, and potassium carbonate $(0.9 \mathrm{~g})$ were dissolved in $\mathrm{N}, \mathrm{N}$-dimethylformamide $(10 \mathrm{~mL})$ and stirred at $60^{\circ} \mathrm{C}$ overnight. Then, $50 \mathrm{~mL}$ of water was added into the reaction mixture to precipitate the product followed by thorough washing with water to give 3,5-dipentadecyloxybenzonitrile with a yield of $61.2 \%$. (Singlet and multiplates were, respectively, abbreviated as s and m. ${ }^{1} \mathrm{H}-\mathrm{NMR}\left(400 \mathrm{MHz}, \mathrm{CDCl}_{3}\right)$ : ppm $\delta 6.73$ (s, 2H), 6.63 (s, 1H), 3.93 (t, 4H, J=4 Hz), 1.77 (t, 4H, J=8 Hz), $1.44(\mathrm{~s}, 4 \mathrm{H}), 1.26(\mathrm{~s}, 44 \mathrm{H}), 0.88(\mathrm{~m}, 6 \mathrm{H}))$ (Figure S1). TRX-20 was synthesized by dissolving $500 \mathrm{mg}$ of 3,5-dipentadecyloxybenzonitrile in $23 \mathrm{~mL}$ of methanol and chloroform (3:20), and injecting dry $\mathrm{HCl}$ gas into the reaction system until saturation. After reaction at ambient temperature overnight, the solvent was evaporated off, and $25 \mathrm{~mL}$ of saturated ammonia in methanol and chloroform (1:4) were added with stirring for $24 \mathrm{~h}$. TRX-20 was obtained as colorless crystals by recrystallization in methanol (yield: 50.4\%). ( $\left.{ }^{1} \mathrm{H}-\mathrm{NMR} \mathrm{CDCl}_{3}, 400 \mathrm{MHz}\right)$ : ppm $\delta 9.80(\mathrm{~s}, 2 \mathrm{H})$, $7.80(\mathrm{~s}, 1 \mathrm{H}), 6.91(\mathrm{~s}, 2 \mathrm{H}), 6.67(\mathrm{~s}, 1 \mathrm{H}), 4.00(\mathrm{t}, 4 \mathrm{H}, \mathrm{J}=4 \mathrm{~Hz})$, 1.77 (t, 4H, J=6), 1.44 (s, 4H), $1.26(\mathrm{~s}, 44 \mathrm{H}), 0.88(\mathrm{~m}, 6 \mathrm{H})$ (Figure S2).

\section{Liposome preparation, labeling, and modification}

According to our previous report, all liposomes were prepared using a lipid film hydration method. ${ }^{18}$ Coumarin6-loaded liposomes (C6-LP) as control were prepared by dissolving C6 (0.5 mg), HSPC, and cholesterol (40 mg, molar ratio of 4:1) in chloroform in a round-bottom flask followed by eliminating the organic solvent under reduced pressure to form the lipid film. After hydration in $5 \mathrm{~mL}$ of phosphate-buffered saline (PBS, $\mathrm{pH}$ 7.2) containing $0.2 \%$ F68, the dispersion was sonicated using a probe ultrasonicator (JY92-II ultrasonic cell crusher processor, Ningbo, China) operating at pulse function (200 watts, 20 hertz; pulse on/ off: $20 \mathrm{~s} / 15 \mathrm{~s} ; 10$ times), and filtered through a $0.2-\mu \mathrm{m}$ filter to obtain liposomes of around $100 \mathrm{~nm}$.

All other liposomes for the study were fabricated using similar procedures and by dissolving the requisite starting materials in the chloroform solution prior to lipid film formation. TRX-20-modified Coumarin-6-loaded liposomes (TRX-C6-LP) were prepared by additionally including TRX-20 at 2.0, 4.0, and $5.0 \mathrm{mg}$ to give liposomes with molar ratios of TRX-20 to total lipids of $6 \%, 11 \%$, and $14 \%$, respectively. Liposomes with 6\%-, 11\%-, and 14\%-TRX-20 to total lipids are abbreviated as $6 \%$-TRX-LP, $11 \%$-TRX-LP, and $14 \%$-TRX-LP, respectively, to ease discussion. Control TRX-20-modified blank liposomes (TRX-LP) were prepared by omitting the use of C6. TP-loaded TRX-LP (TRX-TP-LP) was prepared using $4.0 \mathrm{mg}$ of TRX-20 (11 mole\%) and substituting C6 with TP (4.0 mg), while $\mathrm{PEG}_{5000}$-modified TRX-TP-LP (PEG-TRX-TP-LP) was prepared by also adding $\mathrm{PEG}_{5000}-\mathrm{PE}$ (1.5 mg, $\sim 0.65$ mole $\%$ of the total lipids) besides $4.0 \mathrm{mg}$ each of TRX-20 and TP into the chloroform solution prior to film formation. 
Following the fabrication of the TP-loaded liposomes, any unencapsulated TP was removed by gel filtration, and TP loading concentrations in the TRX-TP-LP and PEGTRX-TP-LP were determined by HPLC after demulsification with methanol.

The particle sizes of the liposomes were determined using a Zetasizer Nano ZS equipment (Malvern Instruments Ltd, Malvern, UK). The morphology of TRX-C6-LP (11\% mole\%) and PEG-TRX-TP-LP was visualized under a transmission electron microscope (TEM, H-7500, Hitachi, Japan) following negative staining with $2 \%$ sodium phosphotungstate solution.

\section{Primary culture of MCs}

MCs were isolated from male SD rats weighing 150-200 g by the sieving method as previously described..$^{19}$ In brief, the kidneys were removed from their capsule and the cortex was separated and cut into fragments $\left(5-10 \mathrm{~mm}^{3}\right)$ followed by mechanical sieving to obtain the glomeruli on 100- and 200-mesh stainless-steel sieves. After enzymatic digestions with collagenase IV $(0.1 \% \mathrm{w} / \mathrm{v})$ at $37^{\circ} \mathrm{C}$ in PBS solution for 20-45 min, the MC suspensions were obtained and cultured in RPMI 1640 medium containing 20\% heat-inactivated fetal bovine serum, $2 \mu \mathrm{g} / \mathrm{mL}$ insulin, $300 \mu \mathrm{g} / \mathrm{L}$ transferrin, $100 \mathrm{U} / \mathrm{mL}$ penicillin, and $100 \mathrm{U} / \mathrm{mL}$ streptomycin at $37^{\circ} \mathrm{C}$ in a humidified 5\% (v/v) $\mathrm{CO}_{2}$ incubator (Thermo Scientific, Marietta, OH, USA). Passages of primary cultures were performed after 3-4 weeks. To eliminate contamination by either epithelial or endothelial cells, experiments were performed on cells obtained after passage 5 .

\section{Cytotoxicity of TRX-LPs}

MCs (passage 6) were seeded onto 96-well plates (Corning, NY, USA) at a density of $1 \times 10^{5}$ cells/well with $200 \mu \mathrm{L}$ of RPMI 1640. After incubation for $24 \mathrm{~h}$, the culture medium was replaced with $200 \mu \mathrm{L}$ of blank liposomes (control) or TRX-LPs at a lipid concentration ranging from 0.01 to $1.0 \mathrm{mg} / \mathrm{mL}$ in RPMI 1640 solutions and the plates were returned to the incubator for another $24 \mathrm{~h}$. The test samples were discarded and the viability of cells was determined by adding $20 \mu \mathrm{L}$ of MTT ( $5 \mathrm{mg} / \mathrm{mL}$ in PBS) and $100 \mu \mathrm{L}$ RPMI 1640 into each well. The medium was aspirated after $2 \mathrm{~h}$ of incubation, and the intracellular formazan dissolved with $150 \mu \mathrm{L}$ dimethyl sulfoxide (DMSO) was quantified by measuring the optical density of each well at $490 \mathrm{~nm}$ (iMark microplate-reader, Bio-rad, CA, USA). Percent cell viability relative to control was plotted as a function of total lipid concentration $(\mu \mathrm{g} / \mathrm{mL})$.

\section{Cellular uptake of TRX-C6-LPs}

MCs were seeded at a density of $2 \times 10^{4}$ cells/well with $2 \mathrm{~mL}$ of RPMI 1640 onto six-well chamber slides (Corning Life Sciences, NY, USA). After 2-3 days of culture, the cells were incubated with C6-LP (control) or TRX-C6-LPs at a lipid concentration of $0.8 \mathrm{mg} / \mathrm{mL}$ for $2 \mathrm{~h}$ in serum-free RPMI 1640 at $37^{\circ} \mathrm{C}$. The cells were then washed with PBS to terminate the uptake process. To visualize the cellular uptake, the cells were fixed in $10 \%$ neutral buffer formalin for $10 \mathrm{~min}$, then counterstained with DAPI for observation under a laser confocal microscope (SP5, Leica, Germany).

\section{Determination of NO and TNF- $\alpha$ production in MCs}

To measure the anti-inflammatory activity of TRX-TP-LP and PEG-TRX-TP-LP, their effects on nitric oxide (NO) and TNF- $\alpha$ production in LPS-stimulated MCs were determined in vitro. ${ }^{20} \mathrm{MCs}$ were seeded at a density of $1 \times 10^{5}$ cells/well in 96-well plates and incubated until confluence. The cells were then exposed to $200 \mu \mathrm{L}$ LPS ( $10 \mu \mathrm{g} / \mathrm{mL}$ in DMEM) alone, or together with TRX-TP-LP or PEG-TRX-TP-LP (TP equivalent concentration of $2.0 \mu \mathrm{g} / \mathrm{mL}$, in DMEM) or TP $(2.0 \mu \mathrm{g} / \mathrm{mL}$ in DMEM with $1 \%$ DMSO). After incubation for $24 \mathrm{~h}, 50 \mu \mathrm{L}$ of supernatant from each well was aspirated into a new 96-well plate for the determination of NO level using nitric oxide assay kits (Jiancheng, Nanjing, China). Similarly, $100 \mu \mathrm{L}$ of supernatant was collected from each well, and the production of TNF- $\alpha$ assessed using a commercial rodent TNF- $\alpha$ enzyme-linked immunosorbent assay kit (Pierce Biotechnology, Rockford, IL, USA). Each treatment was repeated for five separate wells, and the data pooled to obtain the average.

\section{Treatment of nephropathic rats}

SD rats were randomly assigned into normal, control, TP, TRX-TP-LP, and PEG-TRX-TP-LP groups ( $\mathrm{n}=5$ per group). The MN model was established in all except rats in the normal group according to our previous method. ${ }^{11}$ In brief, cationic bovine serum albumin (C-BSA, $1 \mathrm{mg}$ emulsified in $0.5 \mathrm{~mL}$ of Freund's complete adjuvant) was subcutaneously injected into the rats to initiate the immunization. One week later, rats were immunized intravenously with $2.5 \mathrm{mg}$ C-BSA every other day for another 2 weeks to finally establish the $\mathrm{MN}$ rat model. Rats in the normal group followed the same injection schedule but were administered with normal saline instead of C-BSA.

The $\mathrm{MN}$ rats were then administered intravenously with saline (control group), $100 \mu \mathrm{g} / \mathrm{kg}$ TP (TP group) or $\sim 1.6 \mathrm{mg}$ total lipids/kg of TRX-TP-LP (TRX-TP-LP group, 
an equivalent dose of $100 \mu \mathrm{g} / \mathrm{kg} \mathrm{TP})$, or $\sim 1.6 \mathrm{mg}$ total lipids/ $\mathrm{kg}$ of PEG-TRX-TP-LP (PEG-TRX-TP-LP group, an equivalent dose of $100 \mu \mathrm{g} / \mathrm{kg}$ TP) every other day for 2 weeks. Urine samples were collected during the final $24 \mathrm{~h}$ of the experiment from all rats for the measurements of urinary protein $(\mathrm{Up})$ and urinary creatinine (Ucr) levels (Up and creatinine assay kits, Jiancheng, Nanjing, China) to determine the level of proteinuria (ratio of $\mathrm{Up} / \mathrm{Ucr}$ ). Twenty-four hours after the last injection, the animals were killed and blood samples collected from the abdominal aorta were measured for albumin, cholesterol, and creatinine (SCr) levels (7020 automated multiparametric analyzer, Hitachi, Tokyo, Japan).

\section{Data analysis}

Data were analyzed with the SPSS 15.0 statistical package. Multiple comparisons of mean values were performed by one-way analysis of variance with the Fisher's least significant difference test applied for post hoc comparisons at $95 \%$ CI. $P<0.05$ was considered statistically significant.

\section{Results and discussion}

\section{Synthesis and cytotoxicity of TRX-20}

In the study, TRX-20 was synthesized according to the previous reports, in which it was shown that TRX-20 could be used to create a liposomal formulation with an $\mathrm{MC}$ active targeting functionality. ${ }^{16,17}$ The appearance of the signals from the three aromatic protons of the amidine group at ppm 7.80 and 9.80 in the ${ }^{1} \mathrm{H}-\mathrm{NMR}$ spectra indicated the successful synthesis of TRX-20.

Cationic lipids with good affinity to cells have many advantages as nonimmunogenic, nononcogenic, and readily fabricated drug delivery platforms. However, they normally exhibit significant toxicity intrinsically and because of their ability to bind a wide variety of biomolecules, which then limits their biomedical applications. ${ }^{21} \mathrm{~A}$ lipid mixture containing 25 mole $\%$ TRX-20 that was used for condensing DNA to enhance transfection was found to be less cytotoxic against Hep $\mathrm{G} 2$ cells than conventional transfection agents. ${ }^{22}$ However, the effects of TRX-20 on renal cells have yet to be established. In this study, we examined the cytotoxicity of TRX-20 against the MCs to ascertain the appropriate TRX-20 concentration to use that would afford the liposomal formulation a balance of acceptable toxicity and efficacy in the treatment of kidney disease.

TRX-20 was inserted in the bilayer of the liposomes to form 6\%-TRX-LP, $11 \%$-TRX-LP, and 14\%-TRX-LP for the evaluation of cytotoxicity against MCs isolated from SD rats. All three TRX-LPs exhibited concentration-dependent

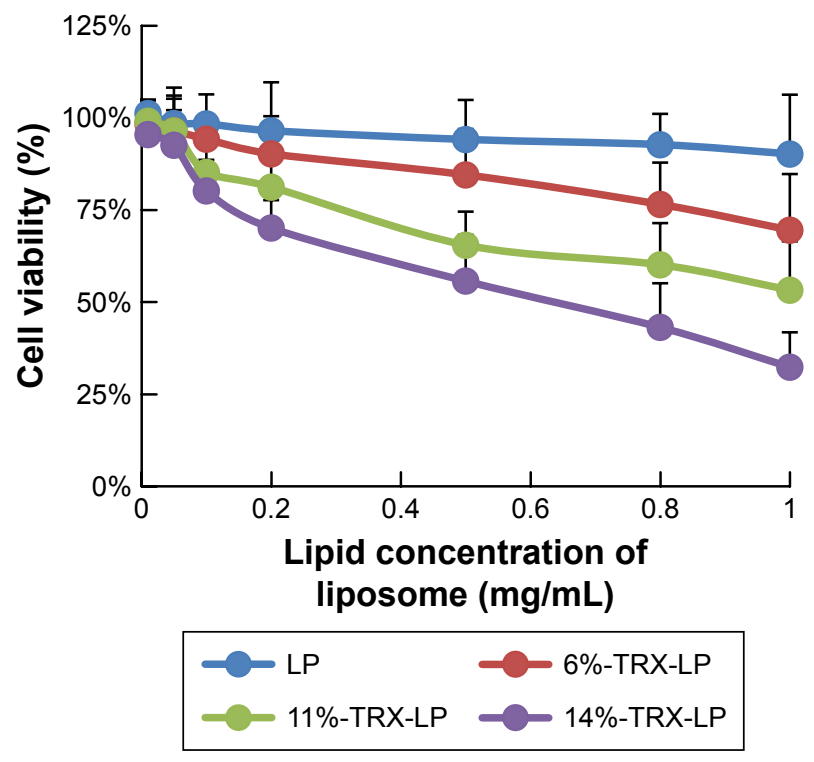

Figure 2 Cytotoxicity of 6\%-TRX-LP, II\%-TRX-LP, and I4\%-TRX-LP against MCs at concentrations equivalent to lipid concentrations between 0.01 and $1.00 \mathrm{mg} / \mathrm{mL}$ for $24 \mathrm{~h}$.

Note: Data represent mean \pm StD $(n=5)$.

Abbreviations: MCs, mesangial cells; LP, lipopolysaccharide; TRX-LP, TRX-20modified liposomes; StD, standard deviation.

toxicity against the MCs at lipid concentration ranging from 0.01 to $1.0 \mathrm{mg} / \mathrm{mL}$, whereas the negative control LPs (without TRX) showed no obvious cytotoxicity after $24 \mathrm{~h}$ incubation (Figure 2). The $\mathrm{IC}_{50}$ values for 6\%-TRX-LP, $11 \%$-TRX-LP, and $14 \%$-TRX-LP were $3.45,1.13$, and $0.55 \mathrm{mg} / \mathrm{mL}$, respectively, indicating that cytotoxicity increased with increasing TRX-20 content. Even when incorporated at a low concentration of 6 mole $\%$ into HSPC, the TRX-20 cationic lipid could provoke cytotoxicity, which was consistent with the results reported by Koiwai et al.

\section{Characteristics of liposomes}

The particle size and zeta potential of the different liposomes are shown in Table 1. C6-LP, which entrapped Coumarin-6 as a trace label, was negatively charged with a zeta potential of $-3.48 \pm 0.21 \mathrm{mV}$ and particle size of $90.7 \pm 1.1 \mathrm{~nm}$.

Table I Characterization of all liposomes

\begin{tabular}{llll}
\hline Sample & Size/nm \pm StD & PDI \pm StD & $\begin{array}{l}\text { Zeta-potential/ } \\
\mathbf{m V} \pm \text { StD }\end{array}$ \\
\hline C6-LP & $90.7 \pm I . I$ & $0.190 \pm 0.005$ & $-3.48 \pm 0.21$ \\
$6 \%-T R X-C 6-L P$ & $101.8 \pm 4.5$ & $0.235 \pm 0.104$ & $8.61 \pm 0.36$ \\
II\%-TRX-C6-LP & $106.4 \pm 3.7$ & $0.216 \pm 0.127$ & $12.2 \pm 0.54$ \\
I4\%-TRX-C6-LP & $110.5 \pm 5.2$ & $0.243 \pm 0.116$ & $15.7 \pm 0.60$ \\
TRX-TP-LP & $107.3 \pm 3.1$ & $0.202 \pm 0.008$ & $12.6 \pm 0.18$ \\
PEG-TRX-TP-LP & $117.9 \pm 1.4$ & $0.191 \pm 0.007$ & $13.4 \pm 0.27$ \\
\hline
\end{tabular}

Abbreviations: C6-LP, Coumarin-6-loaded liposomes; PEG-TRX-TP-LP, TRX-LP with PEG $_{5000}$ co-modification; TP, triptolide; TRX-C6-LP, TRX-20-modified Coumarin-6-loaded liposomes; TRX-TP-LP, triptolide-loaded TRX-LP; StD, standard deviation; PDI, polydispersity index. 
Incorporation of TRX-20 increased the C6-LP diameter and conferred a positive charge, with larger liposomes having higher zeta potential obtained with increasing TRX-20 content.

TP as a model drug was also successfully encapsulated into liposomes to obtain TRX-TP-LP and PEG-TRXTP-LP. TP as a bioactive diterpenoid epoxide isolated from Tripterygium wilfordii Hook $\mathrm{F}$ has promising potential to treat a variety of renal diseases ${ }^{23}$ but its progression into clinical use is hampered by low water solubility (log P 1.494) and poor renal discriminative activity that then led to severe side effects on the circulatory and reproductive systems. ${ }^{24-26}$ To improve its therapeutic potential, much research has been focused on employing appropriate pharmaceutical strategies to attenuate the toxicity and increase the water solubility of TP. ${ }^{11,27-29}$ In this study, the entrapment of TP into $11 \%$-TRX-LP to fabricate TRX-TP-LP did not change the diameter and zeta potential of the liposomes. PEGylation of TRX-TP-LP led to an increase in diameter but had no effect on the zeta potential. The obtained liposome solutions were clear opalescent suspensions without any precipitation of unencapsulated TP. The measured TP concentrations in TRX-TP-LP and PEG-TRX-TP-LP were $0.061 \mathrm{mg} / \mathrm{mg}$ lipid and $0.063 \mathrm{mg} / \mathrm{mg}$ lipid after the removal of unencapsulated TP by gel filtration, respectively.

Under the TEM, 11\%-TRX-C6-LP (Figure 3A) and PEGTRX-TP-LP (Figure 3B) were observed to contain spherical or ellipsoidal vesicles with good dispersity. TEM micrographs of all TRX-20-modified liposomes, with or without C6 and TP loading, consistently showed vesicles of narrow size distribution within the range of 100-120 nm mean diameter, which is beneficial for liposomes traversing through the fenestrated endothelium directly into the mesangial space. ${ }^{5}$ The renal glomeruli have a unique anatomic structure.
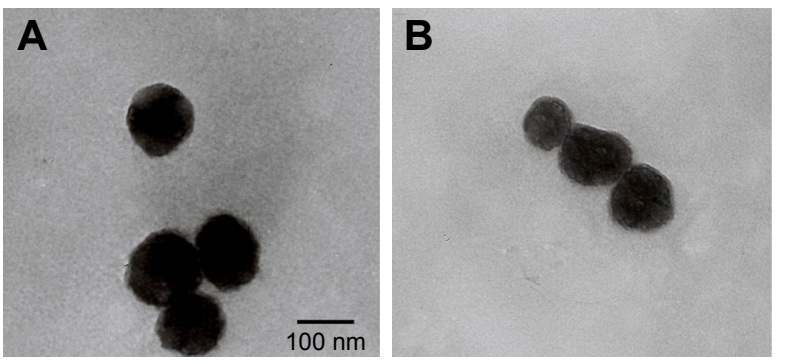

Figure 3 Images of I1\%-TRX-C6-LP (A) and PEG-TRX-TP-LP (B) by negative staining with $2 \%$ sodium phosphotungstate solution under a transmission electron microscope.

Note: Original magnification for both images was $\times 100 \mathrm{k}$.

Abbreviations: C6, Coumarin-6; LP, lipopolysaccharide; PEG-TRX-TP-LP, TRX-LP with PEG $_{5000}$ co-modification; TRX-C6-LP, TRX-20-modified Coumarin-6-loaded liposomes; TRX-LP, TRX-20-modified liposomes.
The glomerular mesangium contains endothelial fenestrations of 130-170 nm, whereas the glomerular basement membrane has much smaller 30-70 nm slit pores. Thus, functionalized liposomes of 100-120 nm are able to pass into the mesangium but not into the glomerular tubules. The resultant entrapment of TRX-LP in the mesangial area is further facilitated by its ability to bind to the MCs in this region.

\section{Specific uptake of TRX-C6-LPs by MCs}

A comparison of the cellular uptake data for C6-LP, 6\%-TRX-C6-LP, 11\%-TRX-C6-LP, and 14\%-TRX-C6-LP (Figure 4) suggests that TRX-20 modification promoted the liposomal uptake by MCs, Higher cellular uptake of the liposomes was observed at increasing TRX-20 content, with 14\%-TRX-C6-LP showing the highest accumulation and evidence of localization in the cytoplasm. Obviously, it is worth noting that the uptake values of the TRX-C6-LPs were much higher than those of C6-LP. However, excessive TRX-20 modification for liposomes showed severe toxicity to viability of $\mathrm{MCs}$ and considerations should be given to balance the toxicity and the uptake efficiency of liposomes. Therefore, TP-loaded liposomes including TRX-TP-LP and PEG-TRX-TP-LP for in vivo therapy were modified with $11 \%$-TRX-20.

\section{Anti-inflammatory activity of TRX-TP-LP and PEG-TRX-TP-LP}

TP is a strong anti-inflammatory agent that has been identified to work in multiple tissues by inhibiting the synthesis and release of inflammatory factors. ${ }^{20}$ In this study, we measured the levels of extracellular NO and TNF- $\alpha$ as they are relevant effector molecules that contribute to glomerular injury in numerous experimental models of glomerulonephritis. ${ }^{30-32}$ Consistent with Zhou's report, the high levels of NO and TNF- $\alpha$ released by the LPS-stimulated MCs were effectively attenuated by co-incubation with TP for $24 \mathrm{~h}$ (Figure 5). TRX-20 has a high affinity for $\mathrm{MCs},{ }^{16}$ and this specificity was exploited in this study to develop renal-targeted therapy of TP. Pegylation of the resultant TRX-TP-LP was also utilized as a means to improve therapeutic efficiency in vivo. Entrapment of TP into the $11 \%$-TRX-LP did not weaken its anti-inflammatory activity, contrary to the expectation that liposomal encapsulation may hinder TP cellular uptake by MCs. ${ }^{33,34}$ PEG chains inserted into the lipid bilayer of TRX-TP-LP also did not significantly decrease anti-inflammatory action. Indeed, TRX-TP-LP and PEG-TRX-TP-LP showed better antiinflammatory actions than naked TP applied at the equivalent concentration in the study $(P<0.05)$, which was probably 

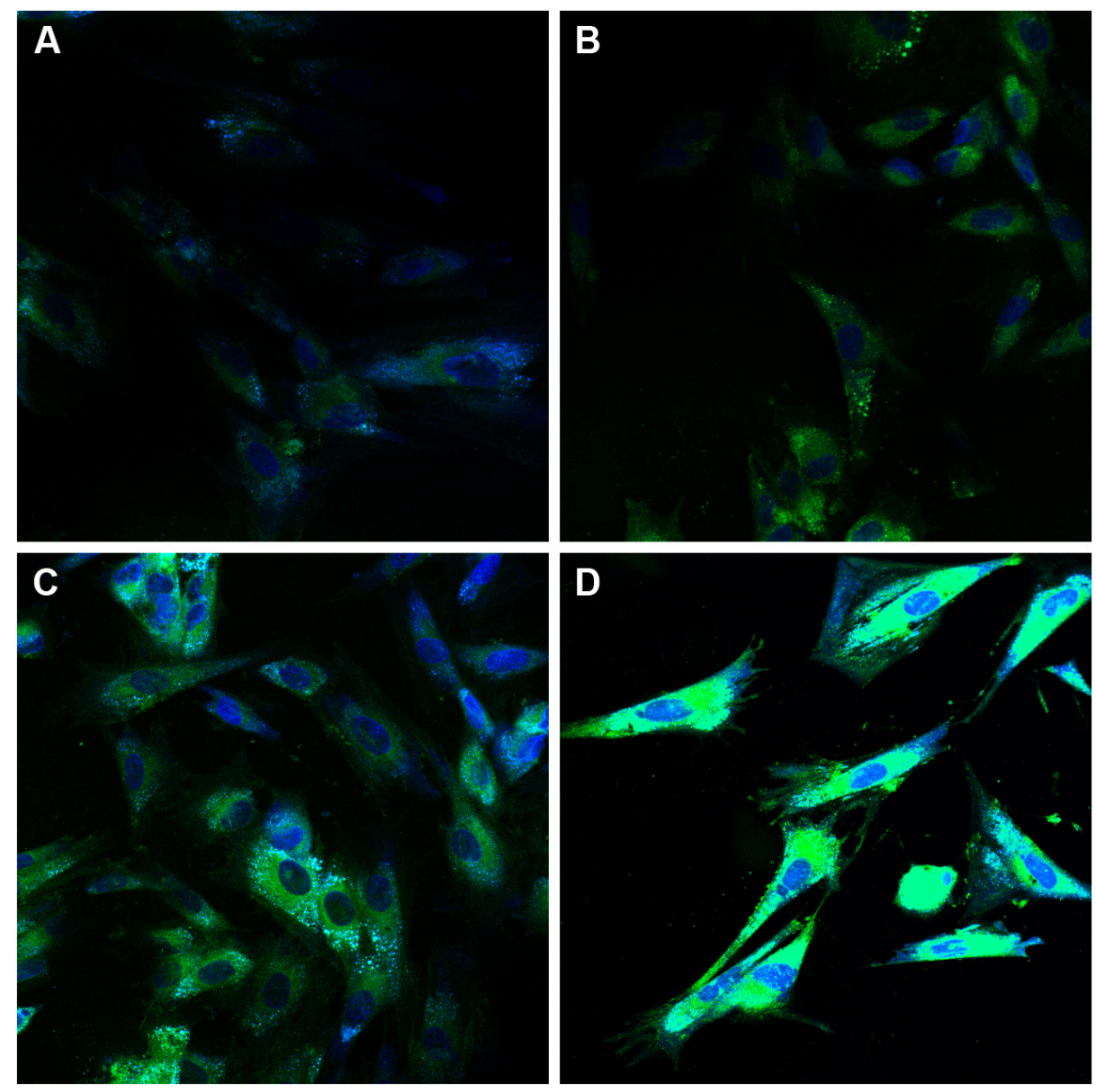

Figure 4 Confocal micrographs showing the uptake by MCs of C6-LP (A), 6\%-TRX-C6-LP (B), II\%-TRX-C6-LP (C), and I4\%-TRX-C6-LP (D) after 24 h incubation (C6: green).

Notes: MCs were incubated with C6-LP (control) or TRX-C6-LPs at a lipid concentration of $0.8 \mathrm{mg} / \mathrm{mL}$ for $2 \mathrm{~h}$ in serum-free RPMI 1640 at $37^{\circ} \mathrm{C}$ and then washed with PBS to terminate the uptake process. After fixation in $10 \%$ neutral buffer formalin, the cells were counterstained with DAPI (blue) for observation under a laser confocal microscope. Magnification $\times 400$.

Abbreviations: C6, Coumarin-6; C6-LP, Coumarin-6-loaded liposomes; DAPI, dihydrochloride; MCs, mesangial cells; PBS, phosphate-buffered saline; TRX-C6-LP, TRX-20-modified Coumarin-6-loaded liposomes.

attributed to the accentuated uptake of TRX-20-modified liposomes by the MCs. As shown in Figure 5, TRX-TP-LP and PEG-TRX-TP-LP significantly inhibited the release of NO (by $71 \%$ and $66 \%$, respectively) and TNF- $\alpha$ (by $52 \%$ and $51 \%$, respectively) that were induced by LPS treatment of the MCs. The results encouraged us to compare the therapeutic efficiency of TRX-TP-LP and PEG-TRX-TP-LP in vivo because the previous studies from Morimoto et al and
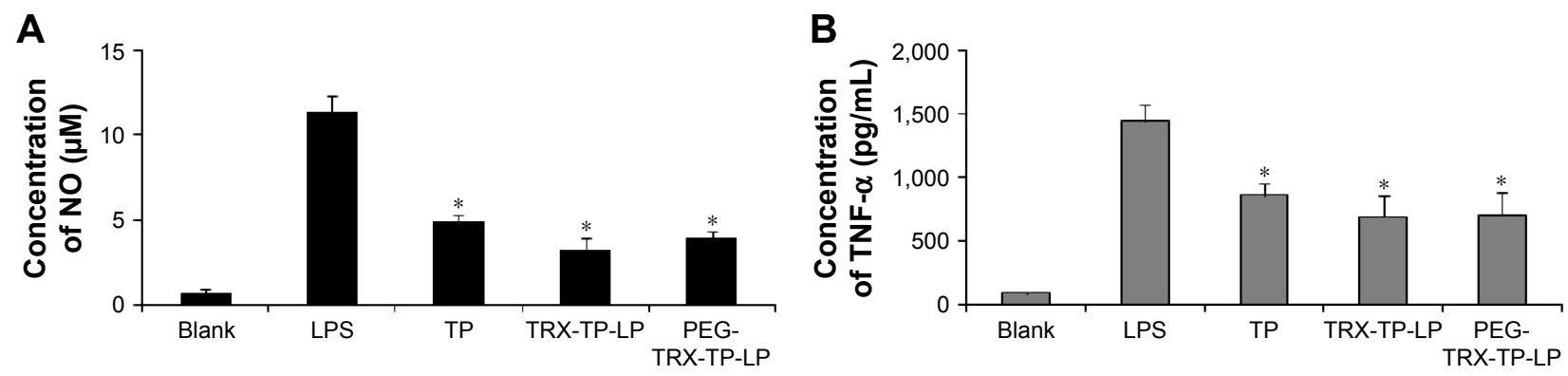

Figure 5 Anti-inflammatory activity of free TP, TRX-TP-LP, and PEG-TRX-TP-LP as measured by an inhibition of NO and TNF- $\alpha$ production by LPS-stimulated MCs. Notes: LPS-stimulated MCs without any treatment served as LPS group while normal MCs as blank. Data represent mean \pm StD ( $n=5) * P<0.05$ versus LPS.

Abbreviations: LPS, lipopolysaccharide; MCs, mesangial cells; NO, nitric oxide; PEG-TRX-TP-LP, TRX-LP with PEG 5000 co-modification; TNF- $\alpha$, tumor necrosis factor- $\alpha$; TP, triptolide; TRX-TP-LP, triptolide-loaded TRX-LP; TRX-LP, TRX-20-modified liposomes; StD, standard deviation. 
Scindia et al reported that only PEGylated liposomes could display better specific accumulation in animal models. ${ }^{15,16}$ It is also worth noting that the anti-inflammatory activity of TP was elicited by applying TRX-TP-LP and PEG-TRXTP-LP at equivalent total lipid concentration of lower than $0.05 \mathrm{mg} / \mathrm{mL}$, which was 22.6 times lower than the $\mathrm{IC}_{50}$ of $11 \%$-TRX-LP, and at which the viability of MCs was 96.51\% (Figure 2).

\section{Renal-targeted therapy}

Modified liposome is an enabling technology for the creation of tissue-/cell-specific therapeutics. The kidney, specifically the glomerulus, is one of the accessible sites for the targeted delivery of drug-loaded liposomes. Renal-targeting therapy is desirable for nephropathy as it limits the immunological events only to the kidneys, thereby reducing systemic side effects. $^{3}$ Morimoto et al were the first to apply TRX-20modified liposomes to renal-targeted therapy, loading the liposomes with prednisolone for the treatment of anti-Thy-1 nephritic rats. ${ }^{16} \mathrm{MCs}$ are the primary targets of immunemediated glomerular diseases and they may also respond to other glomerular injuries that involve the podocytes, endothelial cells, or the glomerular basement membrane. ${ }^{35}$ Therefore, $\mathrm{MC}$ is an ideal targeting site for the treatment of glomerular nephropathy.

In this study, preliminary pharmacodynamics evaluation was performed to ascertain whether TRX-TP-LP and PEGTRX-TP-LP enhanced the renal-targeted pharmacological
A
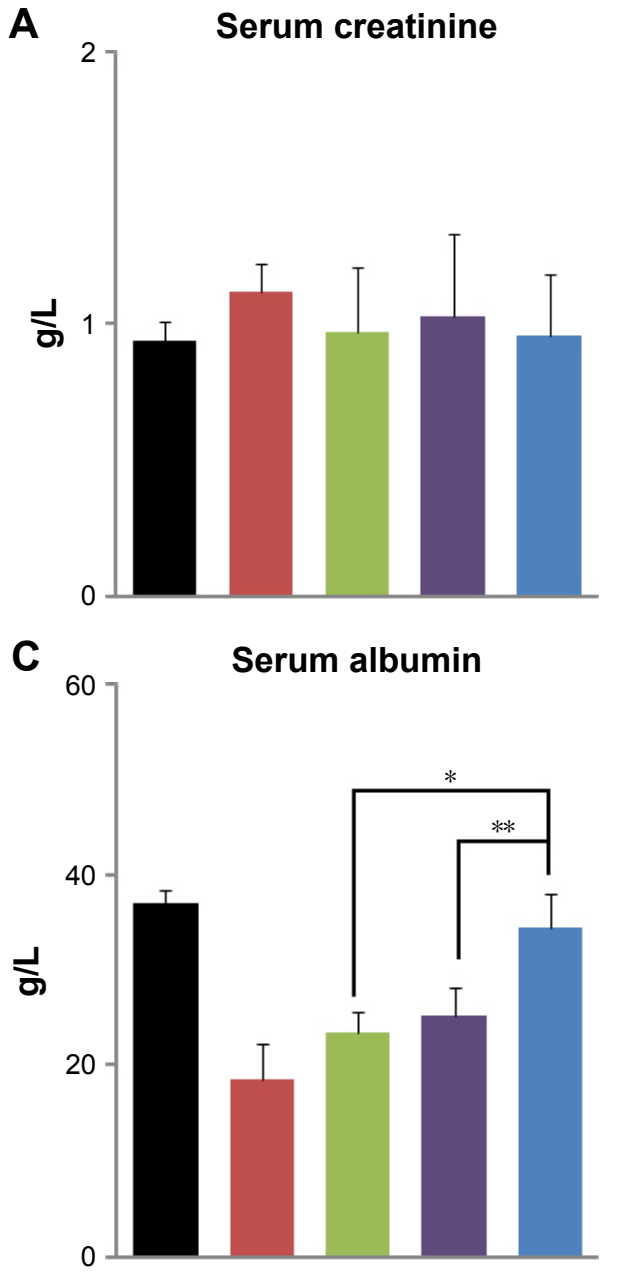

B

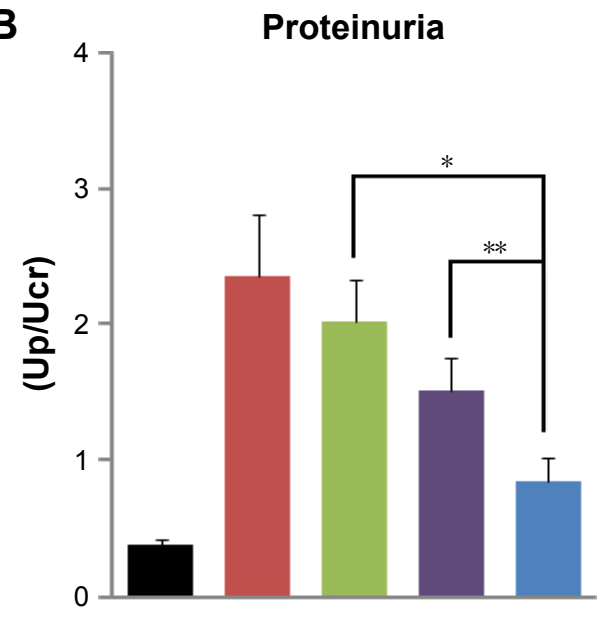

D

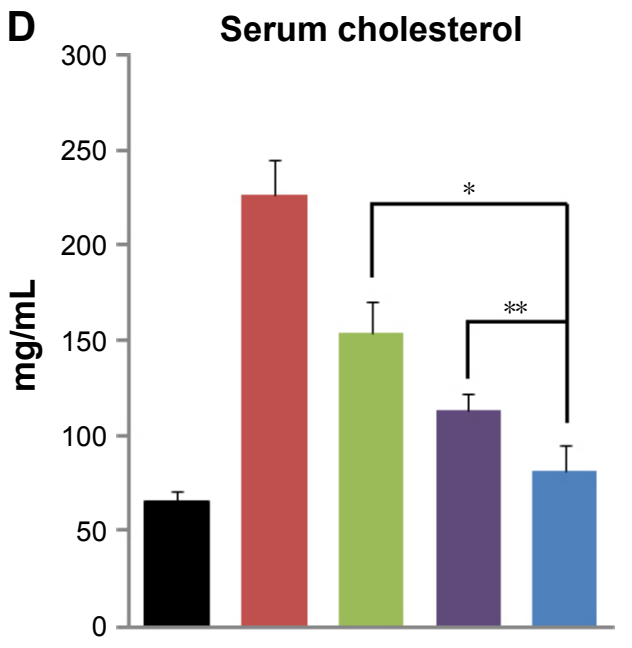

Normal Control $\square$ TP $\square$ TRX-TP-LP $\quad$ PEG-TRX-TP-LP

Figure 6 Biochemical markers of normal SD rats (normal) and SD rats induced with membranous nephropathic (MN) by C-BSA injection over 4 weeks. MN rats received intravenous injections of saline (control), TP solution, or TRX-TP-LP or PEG-TRX-TP-LP dispersions.

Notes: (A) Serum creatinine; (B) proteinuria expressed as the ratio of urinary protein (Up) and urinary creatinine (Ucr) levels; (C) serum albumin; (D) serum cholesterol. Data represent mean $\pm \operatorname{StD}(\mathrm{n}=5)$. $* P<0.05$ versus TP group; $* * P<0.05$ versus TRX-TP-LP group.

Abbreviations: C-BSA, cationic bovine serum albumin; PEG-TRX-TP-LP, TRX-LP with PEG ${ }_{5000}$ co-modification; TP, triptolide; TRX-TP-LP, triptolide-loaded TRX-LP; TRX-LP, TRX-20-modified liposomes; StD, standard deviation. 
activity of TP in vivo. For this purpose, a nephropathic rodent model was established through repeated C-BSA exposure in the SD rats. The nephropathic rodent model follows a similar clinical course and histopathology to human $\mathrm{MN}$. MN is an autoimmune-mediated glomerulonephritis characterized by the presence of diffuse thickening of the glomerular basement membrane and subepithelial in situ immune-complex deposition, and is the most common of nephrotic syndrome in adult humans. Presently available immunosuppressive therapies are not always effective and often present with persistent comorbidities. ${ }^{36}$

The three formulations comprising free TP solution, TRX-TP-LP, and PEG-TRX-TP-LP dispersions were administered intravenously on alternate days over 2 weeks at equivalent TP dose of $100 \mu \mathrm{g} / \mathrm{kg}$ to the rodent $\mathrm{MN}$ model. Compared to rats in the normal group that were not immunized with C-BSA, the $\mathrm{MN}$ rats developed normal SCr levels (Figure 6A) and the characteristic clinical symptoms of overt proteinuria (Figure 6B), hypoalbuminemia (Figure 6C), and hypercholesterolemia (Figure 6D), which were consistent with the biochemical data in our previous study. ${ }^{11}$ Treatment with TP over 2 weeks seemed to alleviate the biochemical abnormalities to some extent, but its efficiency did not match that observed in MN rats treated with TRXTP-LP or PEG-TRX-TP-LP. Treatment with TRX-TP-LP or PEG-TRX-TP-LP effectively attenuated the symptoms and improved biochemical markers including proteinuria, serum cholesterol, and albumin. Relative to the $\mathrm{MN}$ rats in the TRX-TP-LP group, treatment of the MN rats with PEGTRX-TP-LP significantly further reduced the proteinuria and serum cholesterol level, while concomitantly raising the serum albumin level $(P<0.05)$. These three biochemical markers in the PEG-TRX-TP-LP group were brought close to the baseline levels of the normal rats, suggesting that PEGTRX-TP-LP has the potential to reverse the inflammation induced by $\mathrm{C}$-BSA in the SD rats. Histological sections of kidney tissues in each group were also observed under microscope (Figure S3). The histopathological findings supported the biochemical data in advocating the superior efficacy of PEG-TRX-TP-LP.

\section{Conclusion}

Our results clearly demonstrate that the TRX-LPs exhibited TRX-20 concentration-dependent cellular uptake and cytotoxicity against the MCs, with the 14\%-TRX-LP showing highest toxicity and highest accumulation in the cytoplasm of MCs. The incorporation of TP into 11\%-TRX-LP enhances the TP-mediated immune suppression of LPS-stimulated
MCs because of specific binding of the TRX-LP to MCs. Pegylation to give PEG-TRX-TP-LP resulted in further improvement in the anti-inflammatory action of TP in vitro in the LPS-stimulated MCs, and in vivo in the MN rodent model. It can be concluded that TRX- and PEG co-modification of HSPC liposomes could be used for the specific delivery of TP to renal glomeruli for the treatment of $\mathrm{MN}$.

\section{Acknowledgments}

This work was supported by the project (2016M602706) funded by China Postdoctoral Science Foundation and the National Natural Science Foundation of China (81202489).

\section{Disclosure}

The authors report no conflicts of interest in this work.

\section{References}

1. Stenvinkel P. Chronic kidney disease: a public health priority and harbinger of premature cardiovascular disease. J Intern Med. 2010; 268(5):456-467.

2. He XK, Yuan ZX, Wu XJ, Xu CQ, Li WY. Low molecular weight hydroxyethyl chitosan-prednisolone conjugate for renal targeting therapy: synthesis, characterization and in vivo studies. Theranostics. 2012; 2(11):1054-1063.

3. Zhang Z, Zheng Q, Han J, et al. The targeting of 14-succinate triptolide-lysozyme conjugate to proximal renal tubular epithelial cells. Biomaterials. 2009;30(7):1372-1381.

4. Kamada H, Tsutsumi Y, Sato-Kamada K, et al. Synthesis of a poly (vinylpyrrolidone-co-dimethyl maleic anhydride) co-polymer and its application for renal drug targeting. Nat Biotechnol. 2003;21(4): 399-404.

5. Scindia YM, Deshmukh US, Bagavant H. Mesangial pathology in glomerular disease: targets for therapeutic intervention. Adv Drug Deliv Rev. 2010;62(14):1337-1343.

6. Yuan ZX, Sun X, Gong T, Ding H, Fu Y, Zhang ZR. Randomly $50 \%$ $\mathrm{N}$-acetylated low molecular weight chitosan as a novel renal targeting carrier. J Drug Target. 2007;15(4):269-278.

7. Yuan ZX, Zhang ZR, Zhu D, et al. Specific renal uptake of randomly $50 \% \mathrm{~N}$-acetylated low molecular weight chitosan. Mol Pharm. 2009; 6(1):305-314.

8. Lin Y, Li Y, Wang X, Gong T, Zhang L, Sun X. Targeted drug delivery to renal proximal tubule epithelial cells mediated by 2-glucosamine J Control Release. 2013;167(2):148-156.

9. Wang X, Lin Y, Zeng Y, Sun X, Gong T, Zhang Z. Effects of mycophenolic acid-glucosamine conjugates on the base of kidney targeted drug delivery. Int J Pharm. 2013;456(1):223-234.

10. Yuan ZX, He XK, Wu XJ, et al. Peptide fragments of human serum albumin as novel renal targeting carriers. Int J Pharm. 2014;460(1-2): 196-204.

11. Yuan ZX, Wu XJ, Mo J, Wang YL, Xu CQ, Lim LY. Renal targeted delivery of triptolide by conjugation to the fragment peptide of human serum albumin. Eur J Pharm Biopharm. 2015;94:363-371.

12. Maeshima Y. Novel therapeutic approaches for progressive renal disorders by targeting glomerular component mesangial and endothelial cells. Clin Exp Nephrol. 2005;9(4):271-281.

13. Tuffin G, Waelti E, Huwyler J, Hammer C, Marti HP. Immunoliposome targeting to mesangial cells: a promising strategy for specific drug delivery to the kidney. J Am Soc Nephrol. 2005;16(11):3295-3305.

14. Hartner A, Schocklmann H, Prols F, Muller U, Sterzel RB. Alpha8 integrin in glomerular mesangial cells and in experimental glomerulonephritis. Kidney Int. 1999;56(4):1468-1480. 
15. Scindia Y, Deshmukh U, Thimmalapura PR, Bagavant H. Anti-alpha8 integrin immunoliposomes in glomeruli of lupus-susceptible mice: a novel system for delivery of therapeutic agents to the renal glomerulus in systemic lupus erythematosus. Arthritis Rheum. 2008;58(12): 3884-3891.

16. Morimoto K, Kondo M, Kawahara K, et al. Advances in targeting drug delivery to glomerular mesangial cells by long circulating cationic liposomes for the treatment of glomerulonephritis. Pharm Res. 2007;24(5): 946-954.

17. Harigai T, Kondo M, Isozaki M, et al. Preferential binding of polyethylene glycol-coated liposomes containing a novel cationic lipid, TRX-20, to human subendothelial cells via chondroitin sulfate. Pharm Res. 2001; 18(9):1284-1290.

18. Gu J, Yuan Z, Tan R, Zhang X. Isolation of herpetin from herpetospermum seed and hepatoprotective activity of liposomal herpetin against carbon tetrachloride-induced liver injury in mice. Pharmazie. 2015; 70(11):745-752.

19. Gennero I, Fauvel J, Nieto M, et al. Apoptotic effect of sphingosine 1-phosphate and increased sphingosine 1-phosphate hydrolysis on mesangial cells cultured at low cell density. J Biol Chem. 2002;277(15): 12724-12734.

20. Zhou HF, Niu DB, Xue B, et al. Triptolide inhibits TNF-alpha, IL-1 beta and NO production in primary microglial cultures. Neuroreport. 2003;14(7):1091-1095.

21. Lv H, Zhang S, Wang B, Cui S, Yan J. Toxicity of cationic lipids and cationic polymers in gene delivery. J Control Release. 2006;114(1): 100-109.

22. Koiwai K, Tokuhisa K, Karinaga R, et al. Transition from a normal to inverted cylinder for an amidine-bearing lipid/pDNA complex and its excellent transfection. Bioconjug Chem. 2005;16(6):1349-1351.

23. Peng A, Gu Y, Lin SY. Herbal treatment for renal diseases. Ann Acad Med Singapore. 2005;34(1):44-51.

24. Liu J, Jiang Z, Liu L, et al. Triptolide induces adverse effect on reproductive parameters of female sprague-dawley rats. Drug Chem Toxicol. 2011;34(1):1-7.
25. Qi B, Wang X, Zhou Y, et al. A renal-targeted triptolide aminoglycoside (TPAG) conjugate for lowering systemic toxicities of triptolide. Fitoterapia. 2015;103:242-251.

26. Li XJ, Jiang ZZ, Zhang LY. Triptolide: progress on research in pharmacodynamics and toxicology. J Ethnopharmacol. 2014;155(1):67-79.

27. Xu L, Qiu Y, Xu H, Ao W, Lam W, Yang X. Acute and subacute toxicity studies on triptolide and triptolide-loaded polymeric micelles following intravenous administration in rodents. Food Chem Toxicol. 2013;57:371-379.

28. Zhou P, Sun X, Gong T, Zhang Z, Zhang L. Conjugating glucosamine to triptolide to enhance its protective effect against renal ischemia-reperfusion injury and reduce its toxicity. J Drug Target. 2014;22(3):200-210.

29. Xue M, Zhao Y, Li XJ, et al. Comparison of toxicokinetic and tissue distribution of triptolide-loaded solid lipid nanoparticles versus free triptolide in rats. Eur J Pharm Sci. 2012;47(4):713-717.

30. Tipping PG, Leong TW, Holdsworth SR. Tumor necrosis factor production by glomerular macrophages in anti-glomerular basement membrane glomerulonephritis in rabbits. Lab Invest. 1991;65(3):272-279.

31. Ferenbach DA, Ramdas V, Spencer N, et al. Macrophages expressing heme oxygenase-1 improve renal function in ischemia/reperfusion injury. Mol Ther. 2010;18(9):1706-1713.

32. Pfeilschifter J, Eberhardt W, Huwiler A. Nitric oxide and mechanisms of redox signaling. J Am Soc Nephrol. 2003;14(8 Suppl 3):S237-S240.

33. Takara K, Hatakeyama H, Ohga N, Hida K, Harashima H. Design of a dual-ligand system using a specific ligand and cell penetrating peptide, resulting in a synergistic effect on selectivity and cellular uptake. Int $J$ Pharm. 2010;396(1-2):143-148.

34. Cho K, Wang X, Nie S, Chen ZG, Shin DM. Therapeutic nanoparticles for drug delivery in cancer. Clin Cancer Res. 2008;14(5):1310-1316.

35. Abboud HE. Mesangial cell biology. Exp Cell Res. 2012;318(9): 979-985.

36. Wu CC, Lu KC, Chen JS, et al. HO-1 induction ameliorates experimental murine membranous nephropathy: anti-oxidative, anti-apoptotic and immunomodulatory effects. Nephrol Dial Transplant. 2008;23(10): 3082-3090. 


\section{Supplementary materials}

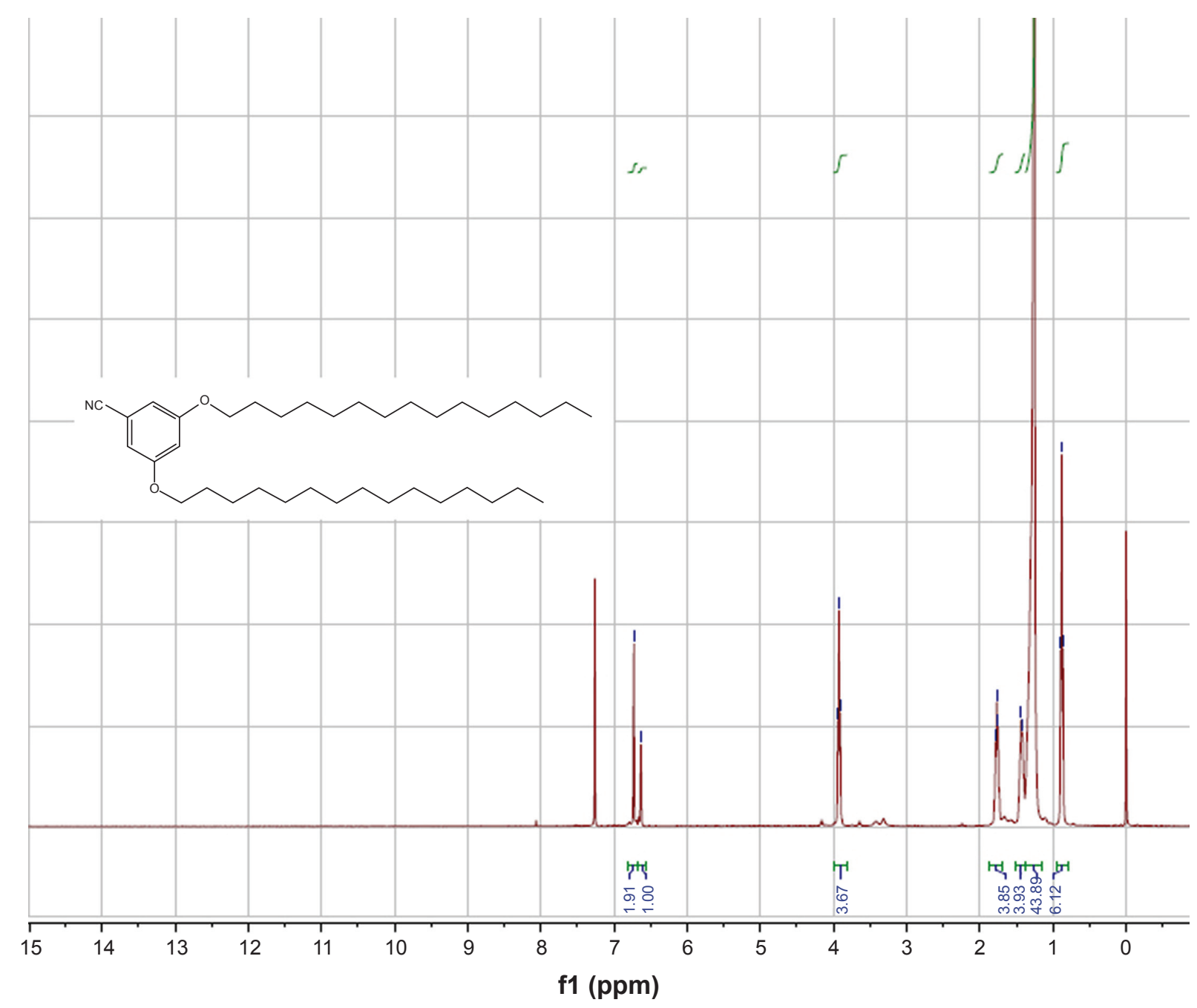

Figure SI Nuclear magnetic resonance spectrum of 3,5-dipentadecyloxybenzonitrile. 


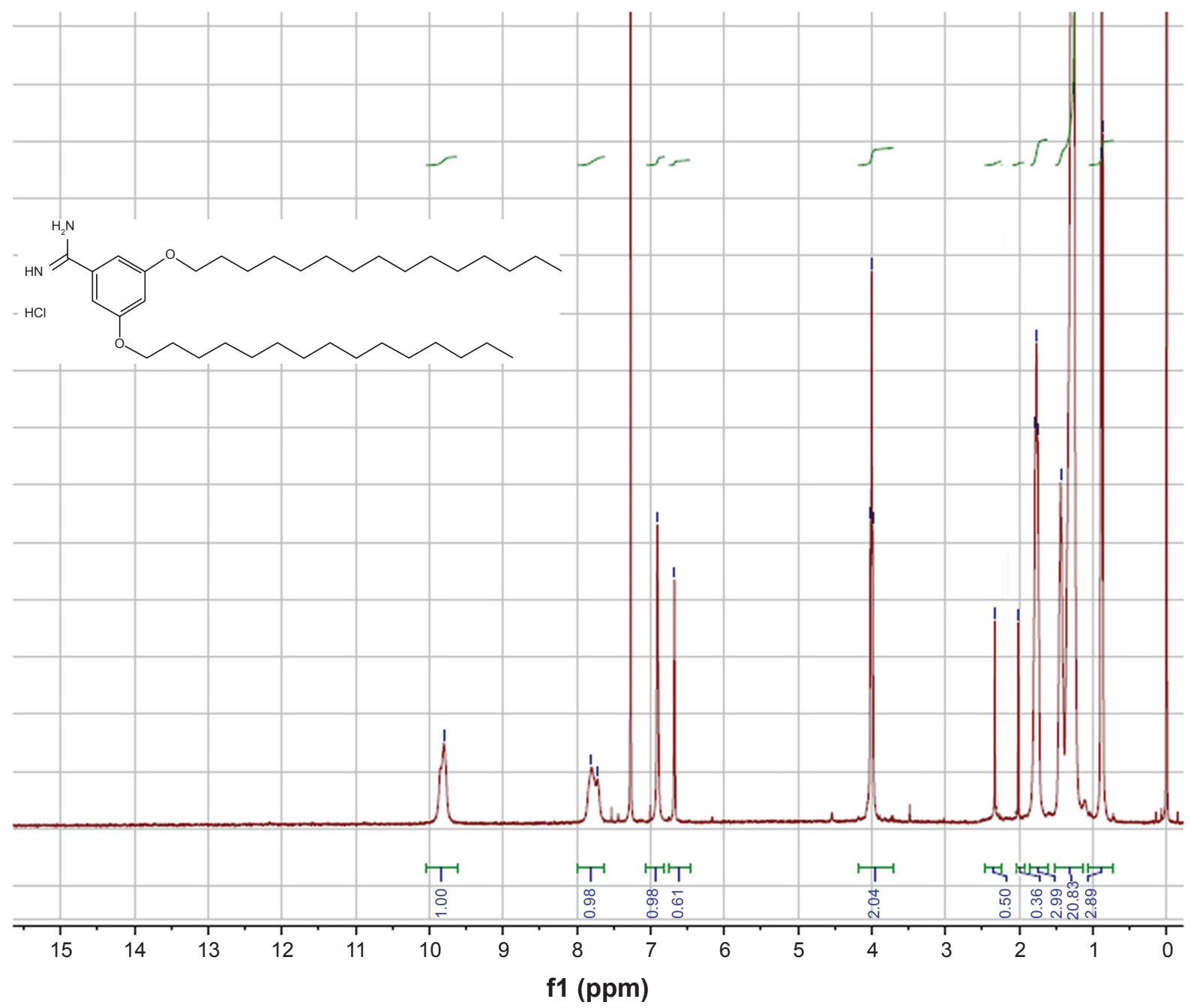

Figure S2 Nuclear magnetic resonance spectrum of 3,5-dipentadecyloxybenzamidine hydrochloride. 

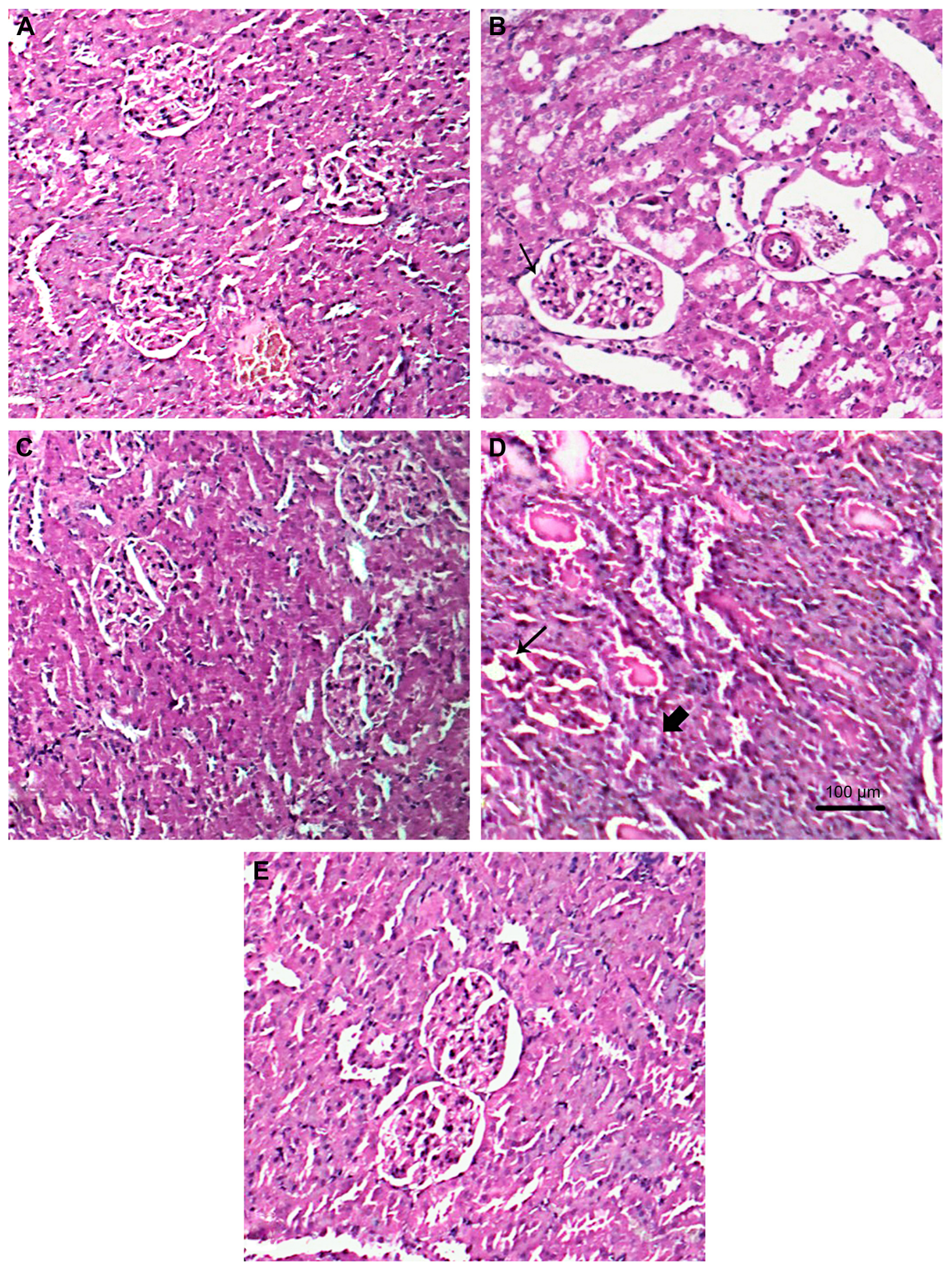

Figure S3 Histological sections of kidney tissues (hematoxylin and eosin staining) harvested on week 6 from euthanized normal Sprague Dawley rats (A) and rats induced with MN by C-BSA injection over 4 weeks, and receiving intravenous injections of control saline solution (B), PEG-TRX-TP-LP dispersions (at equivalent TP dose of $100 \mu \mathrm{g} / \mathrm{kg}$ ) (C), $100 \mu \mathrm{g} / \mathrm{kg}$ TP (D), or TRX-TP-LP dispersions (at equivalent TP dose of $100 \mu \mathrm{g} / \mathrm{kg}$ ) (E). Scale bar $=100 \mu \mathrm{m}$.

Notes: Kidney section for normal group exhibited a histologically normal glomerular architecture (A). In comparison, the kidney section for the control group showed typical nephropathic characteristics of a diffuse basement thickening (thin arrow) of the length of glomerular basement membrane (GBM) (B). After the treatment PEG-TRX-TP-LP (C) or TRX-TP-LP (E), a certain alleviation of pathological changes can be observed, whereas a renal tubular injury (thick arrow) was evident after free TP treatment (D). The histopathological findings supported the biochemical data in advocating the superior efficacy of PEG-TRX-TP-LP.

Abbreviations: C-BSA, cationic bovine serum albumin; LP, lipopolysaccharide; MN, membranous nephropathic; PEG-TRX-TP-LP, TRX-LP with PEG ${ }_{5000}$ co-modification; TP, triptolide; TRX-TP-LP, triptolide-loaded TRX-LP; TRX-LP, TRX-20-modified liposomes. 


\section{Publish your work in this journal}

The International Journal of Nanomedicine is an international, peerreviewed journal focusing on the application of nanotechnology in diagnostics, therapeutics, and drug delivery systems throughout the biomedical field. This journal is indexed on PubMed Central, MedLine, CAS, SciSearch $\AA$, Current Contents $\AA /$ Clinical Medicine,

Journal Citation Reports/Science Edition, EMBase, Scopus and the Elsevier Bibliographic databases. The manuscript management system is completely online and includes a very quick and fair peer-review system, which is all easy to use. Visit http://www.dovepress.com/ testimonials.php to read real quotes from published authors.

Submit your manuscript here: http://www.dovepress.com/international-journal-of-nanomedicine-journal 